\title{
Automatic presentations and semigroup constructions
}

\author{
Alan J. Cain ${ }^{1}$, Graham Oliver ${ }^{2}$, Nik Ruškuc ${ }^{3}$, Richard M. Thomas ${ }^{4}$ \\ ${ }^{1,3}$ School of Mathematics and Statistics, University of St Andrews, \\ North Haugh, St Andrews, Fife KY16 9SS, United Kingdom. \\ ${ }^{2,4}$ Department of Computer Science, University of Leicester, \\ University Road, Leicester, LE1 7RH, United Kingdom \\ 19alanc@mcs.st-andrews.ac.uk, ${ }^{2}$ G.Oliver@mcs.le.ac.uk, \\ ${ }^{3}$ nik@mcs.st-andrews.ac.uk, ${ }^{4}$ rmt@mcs.le.ac.uk
}

\begin{abstract}
An automatic presentation for a relational structure is, informally, an abstract representation of the elements of that structure by means of a regular language such that the relations can all be recognized by finite automata. A structure admitting an automatic presentation is said to be FA-presentable. This paper studies the interaction of automatic presentations and certain semigroup constructions, namely: direct products, free products, finite Rees index extensions and subsemigroups, strong semilattices of semigroups, Rees matrix semigroups, Bruck-Reilly extensions, zero-direct unions, semidirect products, wreath products, ideals, and quotient semigroups. For each case, the closure of the class of FA-presentable semigroups under that construction is considered, as is the question of whether the FA-presentability of the semigroup obtained from such a construction implies the FApresentability of the original semigroup $[\mathrm{s}]$. Classifications are also given of the FA-presentable finitely generated Clifford semigroups, completely simple semigroups, and completely 0-simple semigroups.
\end{abstract}

\section{Introduction}

Automatic presentations ultimately stem from computer scientists' need to extend finite model theory to finite descriptions of infinite structures. In moving to general infinite structures, decidability is of course lost. There has therefore been an effort to find classes of infinite structures admitting at least a modicum of decidability. Khoussainov \& Nerode [15] introduced the concept of an automatic presentation for a relational structure, which (loosely) consists of a regular language representing the elements of the structure in such a way that the relations of the structure can be recognized 
by synchronous finite state automata. Any FA-presentable structure - that is, any structure admitting an automatic presentation - has decidable firstorder theory. This is an important motivation for studying FA-presentable structures. The theory of automatic presentations continues to be developed; see, for example, $[2,3,4,18]$.

A common theme in the research thus far on automatic presentations has been the classification of those structures, within particular species, that admit automatic presentations. The second and fourth authors showed that a finitely generated group admits an automatic presentations if and only if it is virtually abelian (see Theorem 2.8 below). The authors together proved that a finitely generated cancellative semigroup admits an automatic presentation if and only if it embeds into a virtually abelian group [6, 7].

The present paper studies the interaction of automatic presentations and certain semigroup constructions, namely: direct products, free products, finite Rees index extensions and subsemigroups, strong semilattices of semigroups, Rees matrix semigroups, Bruck-Reilly extensions, zero-direct unions, semidirect products, wreath products, ideals, and quotient semigroups. For each case, the closure of the class of FA-presentable semigroups under that construction is considered, as is the question of whether the FApresentability of the semigroup obtained from such a construction implies the FA-presentability of the original semigroup[s].

This study of constructions leads to new characterizations of FA-presentable semigroups of certain classes:

- The finitely generated FA-presentable Clifford semigroups are precisely the finitely generated strong semilattices of virtually abelian groups (Theorem 7.7).

- The finitely generated FA-presentable completely simple and completely 0-simple semigroups are precisely those arising as Rees matrix semigroups over virtually abelian groups (Theorem 8.6 and Corollary 8.7).

\section{Automatic Presentations}

Definition 2.1. Let $L$ be a regular language over a finite alphabet $A$. Define, for $n \in \mathbb{N}$,

$$
L^{n}=\left\{\left(w_{1}, \ldots, w_{n}\right): w_{i} \in L \text { for } i=1, \ldots, n\right\} .
$$


Let $\$$ be a new symbol not in $A$. The mapping conv : $\left(A^{*}\right)^{n} \rightarrow\left((A \cup\{\$\})^{n}\right)^{*}$ is defined as follows. Suppose

$$
\begin{aligned}
& w_{1}=w_{1,1} w_{1,2} \cdots w_{1, m_{1}}, \\
& w_{2}=w_{2,1} w_{2,2} \cdots w_{2, m_{2}}, \\
& \vdots \\
& w_{n}=w_{n, 1} w_{n, 2} \cdots w_{n, m_{n}},
\end{aligned}
$$

where $w_{i, j} \in A$. Then $\operatorname{conv}\left(w_{1}, \ldots, w_{n}\right)$ is defined to be

$$
\left(w_{1,1}, w_{2,1}, \ldots, w_{n, 1}\right)\left(w_{1,2}, w_{2,2}, \ldots, w_{n, 2}\right) \cdots\left(w_{1, m}, w_{2, m}, \ldots, w_{n, m}\right),
$$

where $m=\max \left\{m_{i}: i=1, \ldots, n\right\}$ and with $w_{i, j}=\$$ whenever $j>m_{i}$.

Observe that the mapping conv maps an $n$-tuple of words to a word of $n$-tuples.

Definition 2.2. Let $A$ be a finite alphabet, and let $R \subseteq\left(A^{*}\right)^{n}$ be a relation on $A^{*}$. Then $R$ is said to be regular if

$$
\left\{\operatorname{conv}\left(w_{1}, \ldots, w_{n}\right):\left(w_{1}, \ldots, w_{n}\right) \in R\right\}
$$

is a regular language over $(A \cup\{\$\})^{n}$.

Definition 2.3. Let $\mathcal{S}=\left(S, R_{1}, \ldots, R_{n}\right)$ be a relational structure. Let $L$ be a regular language over a finite alphabet $A$, and let $\phi: L \rightarrow S$ be a surjective mapping. Then $(L, \phi)$ is an automatic presentation for $\mathcal{S}$ if:

1. the relation $L==\left\{\left(w_{1}, w_{2}\right) \in L^{2}: w_{1} \phi=w_{2} \phi\right\}$ is regular, and

2. for each relation $R_{i}$ of arity $r_{i}$, the relation

$$
L_{R_{i}}=\left\{\left(w_{1}, w_{2}, \ldots, w_{r_{i}}\right) \in L^{r_{i}}: R\left(w_{1} \phi, \ldots, w_{r_{i}} \phi\right)\right\}
$$

is regular.

A semigroup can be viewed as a relational structure, with the binary operation $\circ$ becoming a ternary relation. The following definition simply restates the preceding one in the special case when the structure is a semigroup:

Definition 2.4. Let $S$ be a semigroup. Let $L$ be a regular language over a finite alphabet $A$, and let $\phi: L \rightarrow S$ be a surjective mapping. Then $(L, \phi)$ is an automatic presentation for $S$ if the relations

$$
L_{=}=\left\{\left(w_{1}, w_{2}\right) \in L^{2}: w_{1} \phi=w_{2} \phi\right\}
$$

and

$$
L_{\circ}=\left\{\left(w_{1}, w_{2}, w_{3}\right) \in L^{3}:\left(w_{1} \phi\right)\left(w_{2} \phi\right)=w_{3} \phi\right\}
$$

are regular. 
Definition 2.5. Let $(L, \phi)$ be an automatic presentation for a structure. Then $(L, \phi)$ is a binary automatic presentation if the language $L$ is over a two-letter alphabet; and it is an injective automatic presentation if the mapping $\phi$ is injective (so that every element of the structure has exactly one representative in $L$ ).

Proposition 2.6 ([2, Lemma 3.3 \& Theorem 3.4]). Any structure that admits an automatic presentation admits an injective binary automatic presentation.

The fact that a tuple of elements $\left(a_{1}, \ldots, a_{n}\right)$ of a structure $S$ satisfies a first-order formula $\theta\left(x_{1}, \ldots, x_{n}\right)$ is denoted $S \models \theta\left(a_{1}, \ldots, a_{n}\right)$.

Proposition $2.7([15])$. Let $\mathcal{S}$ be a structure with an automatic presentation. For every first-order formula $\theta\left(x_{1}, \ldots, x_{n}\right)$ over the structure there is an automaton which accepts $\left(w_{1}, \ldots, w_{n}\right)$ if and only if $S \models \theta\left(w_{1} \phi, \ldots, w_{n} \phi\right)$. Moreover, this automaton may be effectively constructed.

Theorem $2.8([16])$. A finitely generated group admits an automatic presentation if and only if it is virtually abelian. In particular, a group $G$ with a subgroup $\mathbb{Z}^{n}$ of index $l$ admits an automatic presentation $(L, \phi)$, where $L$ is the language of words

$$
g_{i} \operatorname{conv}\left(\varepsilon_{1} z_{1}, \ldots, \varepsilon_{n} z_{n}\right),
$$

where $\varepsilon_{i} \in\{+,-\}, z_{i}$ is a natural number in reverse binary notation, $g_{1}, \ldots, g_{l}$ are representives of the cosets of $\mathbb{Z}^{n}$ in $G$, with $\phi: L \rightarrow G$ being defined in the natural way:

$$
\phi\left(g_{i} \operatorname{conv}\left(\varepsilon_{1} z_{1}, \ldots, \varepsilon_{n} z_{n}\right)\right)=g_{i}\left(\varepsilon_{1} z_{1}, \ldots, \varepsilon_{n} z_{n}\right) .
$$

Theorem 2.9. Any finitely generated subsemigroup of a semigroup admitting an automatic presentation has polynomial growth. In particular, any FA-presentable finitely generated semigroup has polynomial growth.

Proof. This result was proved for groups in [16]; the proof immediately generalizes to semigroups (see [7]).

\section{Direct products}

The class of FA-presentable semigroups, like the class of FA-presentable structures, is closed under forming direct products [2, Corollary 5.26]. A natural question is whether the converse holds: if $S$ and $T$ are semigroups and $S \times T$ is FA-presentable, must the two direct factors $S$ and $T$ themselves be FA-presentable? This section exhibits a counterexample to show that this does not hold. 
Before embarking on the counterexample, notice that it is particularly important in light of the long-standing open problem of whether automaticity in the sense of Epstein et al. [11] and Campbell et al. [9] is preserved on passing to direct factors; this question is open even in the restricted case of groups.

Example 3.1. Let $Y$ be a non-recursively enumerable subset of the natural numbers. For each $y \in Y$, let $P_{y}$ be a set of $y$ elements, with all the sets $P_{y}$ being pairwise disjoint. Let $e$ and $z$ be new elements. Let

$$
S=\{e, z\} \cup \bigcup_{y \in Y} P_{y} .
$$

Define a multiplication on $S$ by

$$
\begin{aligned}
& u v= \begin{cases}e & \text { if } u, v \in P_{y} \text { for some } y \in Y \\
z & \text { if } u \in P_{x} \text { and } v \in P_{y} \text { for } x, y \in Y \text { with } x \neq y\end{cases} \\
& u e=e u=u z=z u=z \text { for } u \in S
\end{aligned}
$$

(It is easy to check that this multiplication is associative and so $S$ is a semigroup.) Then $S$ is not FA-presentable, for if it were, one could enumerate the set $Y$ : to determine whether $k$ lies in $Y$, one would check whether the set

$$
\begin{aligned}
\left\{\left(x_{1}, \ldots, x_{k}\right):(\right. & \forall i, j \in\{1, \ldots, k\})\left(\left(i \neq j \Longrightarrow x_{i} \neq x_{j}\right) \wedge\left(x_{i} x_{j}=e\right)\right) \\
& \left.\wedge(\forall y \in S)\left(\left(y x_{1}=e\right) \Longrightarrow(\exists k \in\{1, \ldots, k\})\left(y=x_{k}\right)\right)\right\}
\end{aligned}
$$

is non-empty. (That is, check whether there is a set of $k$ distinct elements with pairwise product $e$ and that this set does lie inside a larger such set.)

Let $T$ be a countable null semigroup, with all products being equal to $f \in T$. Then $T$ is FA-presentable.

Observe that $S \times T$ consists of elements

$$
(e, f),(z, f),(p, f),(e, t),(z, t),(p, t),
$$

where $t \in T-\{f\}$ and $p \in P_{y}$ for some $y \in Y$.

Suppose $y_{0}, y_{1}, \ldots$ and $t_{0}, t_{1} \ldots$ are the elements of $Y$ and $T$ respectively, listed in some fixed order with $t_{0}=f$. For each $\left.i \in \mathbb{N} \cup\{0\}\right\}$, let $\left\{q_{i, j}: j \in\right.$ $\mathbb{N} \cup\{0\}\}$ consist of the [countably many] elements $(p, t)$ where $p \in P_{y_{i}}$ and $t \in T$. Let $a$ and $b$ be symbols and let

$$
L=\{\operatorname{conv}(a, j), \operatorname{conv}(b, j), \operatorname{conv}(i, j): i, j \in \mathbb{N} \cup\{0\}\},
$$

where $i$ and $j$ are understood to be in reverse binary notation. Notice that $L$ is a regular language. Define $\phi: L \rightarrow S \times T$ by

$$
\begin{aligned}
& \operatorname{conv}(a, j) \mapsto\left(e, t_{j}\right) \\
& \operatorname{conv}(b, j) \mapsto\left(z, t_{j}\right) \\
& \operatorname{conv}(i, j) \mapsto q_{i, j} .
\end{aligned}
$$


The map $\phi$ is bijective, so $L_{=}=\{(w, w): w \in L\}$. Moreover,

$$
\begin{aligned}
L_{\circ} & =\{(\operatorname{conv}(a, j), \operatorname{conv}(a, k), \operatorname{conv}(b, 0)): j, k \in \mathbb{N} \cup\{0\}\} \\
& \cup\{(\operatorname{conv}(a, j), \operatorname{conv}(b, k), \operatorname{conv}(b, 0)): j, k \in \mathbb{N} \cup\{0\}\} \\
& \cup\{(\operatorname{conv}(a, j), \operatorname{conv}(h, k), \operatorname{conv}(b, 0)): h, j, k \in \mathbb{N} \cup\{0\}\} \\
& \cup\{(\operatorname{conv}(b, j), \operatorname{conv}(a, k), \operatorname{conv}(b, 0)): j, k \in \mathbb{N} \cup\{0\}\} \\
& \cup\{(\operatorname{conv}(b, j), \operatorname{conv}(b, k), \operatorname{conv}(b, 0)): j, k \in \mathbb{N} \cup\{0\}\} \\
& \cup\{(\operatorname{conv}(b, j), \operatorname{conv}(h, k), \operatorname{conv}(b, 0)): h, j, k \in \mathbb{N} \cup\{0\}\} \\
& \cup\{(\operatorname{conv}(i, j), \operatorname{conv}(a, k), \operatorname{conv}(b, 0)): j, k \in \mathbb{N} \cup\{0\}\} \\
& \cup\{(\operatorname{conv}(i, j), \operatorname{conv}(b, k), \operatorname{conv}(b, 0)): i, j, k \in \mathbb{N} \cup\{0\}\} \\
& \cup\{(\operatorname{conv}(i, j), \operatorname{conv}(h, k), \operatorname{conv}(a, 0)): h, i, j, k \in \mathbb{N} \cup\{0\}, h=i\} \\
& \cup\{(\operatorname{conv}(i, j), \operatorname{conv}(h, k), \operatorname{conv}(b, 0)): h, i, j, k \in \mathbb{N} \cup\{0\}, h \neq i\} .
\end{aligned}
$$

The relation $L_{\circ}$ is regular, since the only checking the automaton has to perform is when the first two tracks on the input tape are labelled by $\operatorname{conv}(i, j)$ and $\operatorname{conv}(h, k)$, when it must check whether $h$ and $i$ coincide.

Thus $(L, \phi)$ is an automatic presentation for $S \times T$. Thus $S \times T$ is an example of an FA-presentable direct product with a direct factor $S$ that is not FA-presentable.

In the above example, the countable null semigroup $T$ is FA-presentable. This raises the following question:

Question 3.2. Do there exist semigroups $S$ and $T$ such that $S \times T$ is FApresentable but neither $S$ nor $T$ are?

\section{Free products}

The present section characterizes those semigroup and monoid free products that are FA-presentable. A semigroup free product is only FA-presentable in one very restricted case:

Proposition 4.1. The semigroup free product of two semigroups $S$ and $T$ is FA-presentable if and only if $S$ and $T$ are trivial.

Proof. Suppose $S$ is non-trivial. Let $s_{1}$ and $s_{2}$ be distinct elements of $S$ and let $t \in T$. Then $\left\{s_{1} t, s_{2} t\right\}$ generates a free subsemigroup of $S * T$, which contradicts the fact that every finitely generated subsemigroup of an FA-presentable semigroup has polynomial growth (Theorem 2.9).

Now suppose $S=\{s\}$ and $T=\{t\}$ are both trivial. Then every element of $S * T$ is an alternating product of symbols $s$ and $t$ and is uniquely determined by the leftmost symbol and the length of the product. So let

$$
L=\left\{x n: x \in\{s, t\}, n \in\{0,1\}^{+} 1\right\},
$$


and define $\phi: L \rightarrow S * T$ be letting $(x n) \phi$ be the element of $S * T$ (viewed as a set of alternating products) that starts with $x$ and has length equal to the number represented in reverse binary notation by $n$.

To see that $(L, \phi)$ is an automatic presentation for $S * T$, reason as follows: Every element of $S * T$ has a unique representative in $L$, so the equality relation is simply the diagonal relation $\{(w, w): w \in L\}$. The multiplication relation is

$$
\begin{aligned}
\{(x n, y m, x p) & :((x=y) \wedge(n \equiv 0 \bmod 2) \wedge(p=n+m)) \\
& \vee((x=y) \wedge(n \equiv 1 \bmod 2) \wedge(p=n+m-1)) \\
& \vee((x \neq y) \wedge(n \equiv 0 \bmod 2) \wedge(p=n+m-1)) \\
& \vee((x \neq y) \wedge(n \equiv 1 \bmod 2) \wedge(p=n+m))\},
\end{aligned}
$$

since $(x n) \phi$ ends with $x$ if and only if $n$ is odd. Since addition of numbers in reverse binary notation and checking such numbers for divisibility by 2 can be carried out by an automaton, this relation is regular.

For monoid free products - where the identities of the two monoids are amalgamated - there is marginally more freedomn:

Proposition 4.2. The monoid free product of two monoids $S$ and $T$ is FA-presentable if and only if one of the following cases holds:

1. $S$ is FA-presentable and $T$ is trivial, or vice versa;

2. $S$ and $T$ both contain exactly two elements.

Proof. If $T$ is trivial then $S * T$ is isomorphic to $S$. So assume both $S$ and $T$ are non-trivial.

Suppose $S$ contains at least three elements. Let $s_{1}$ and $s_{2}$ be non-identity elements of $S$ and let $t$ be a non-identity element of $T$. Then $\left\{s_{1} t, s_{2} t\right\}$ generates a free subsemigroup of $S * T$, which is a contradiction, as in the proof of the preceding result.

Now suppose that $S$ and $T$ each have two elements. Then every element of $S * T$ is either the identity or an alternating product of the non-identity elements $s$ of $S$ and $t$ of $T$ which is determined by its leftmost multiplicand and its length. As in the previous proof, let

$$
L=\{e\} \cup\left\{x n: x \in\{s, t\}, n \in\{0,1\}^{+} 1\right\},
$$

and define $\phi: L \rightarrow S * T$ by letting $e \phi$ be the identity and letting $(x n) \phi$ be the element of $S * T$ (viewed as a set of alternating products) that starts with $x$ has length equal to the number represented in reverse binary notation by $n$.

Reasoning similar to the preceding proof shows that $(L, \phi)$ is an automatic structure: the only difference is that there are several cases, depending on whether $s^{2}=s$ or $s^{2}=1_{S}$ and similarly for $t^{2}$. 
Notice that the characterization of FA-presentable monoid free products also characterizes FA-presentable free products of groups.

\section{A noteworthy example}

This section exhibits a particular FA-presentable semilattice which is, in some sense, an extreme example: several semigroup constructions based on this semilattice turn out not to be FA-presentable. More simply, this semilattice is the starting-point for various counterexamples that show the class of FA-presentable semigroups is not closed under certain constructions.

Recall that a semilattice $(S, \leq)$ forms a commutative semigroup of idempotents $(S, \circ)$, where $s \circ t$ is defined to be the greatest lower bound of $\{s, t\}$. That is, $s \circ t$ is the [necessarily unique] element $x \in S$ such that

$$
(x \leq s) \wedge(x \leq t) \wedge(\forall y \in S)(((y \leq s) \wedge(y \leq t)) \Longrightarrow(y \leq x)) .
$$

Therefore $\circ$ is first-order definable over $(S, \leq)$ and similarly $\leq$ is first-order definable over $(S, \circ)$, since

$$
s \leq t \Longleftrightarrow(s \circ t=s) .
$$

Ergo, for the purposes of automatic presentations, one is free to view a semilattice either as an ordered set or as a semigroup.

Let $S$ be the set

$$
\{0\} \cup \bigcup_{i \in \mathbb{N}} M_{i}
$$

where $M_{i}=\left\{m_{i, j}: 1 \leq j \leq i\right\}$ for each $i \in \mathbb{N}$. Let $\preceq$ be the following relation on $S$ :

$$
\begin{aligned}
& 0 \preceq m_{i, j} \quad \text { for all } i, j \in \mathbb{N} \text { with } j \leq i \\
& m_{i, j} \preceq m_{i, k} \quad \text { for all } i, j, k \in \mathbb{N} \text { with } j \leq k \leq i \text {. }
\end{aligned}
$$

The relation $\preceq$ is a partial order on $S$. Furthermore, any two elements of $S$ have a greatest lower bound under $\preceq$. Thus $S$ is a semilattice. The Hasse diagram of $(S, \preceq)$ is shown in Figure 1 .

Proposition 5.1. The semilattice $(S, \preceq)$ admits an automatic presentation.

Proof. Let $L$ be the language

$$
\{z\} \cup\{\operatorname{conv}(i, j): i, j \in \mathbb{N}, j \leq i\},
$$

where the natural numbers $i$ and $j$ are understood to be expressed in reverse binary notation; an automaton can ensure that $j$ does not exceed $i$. The map $\phi: L \rightarrow S$ is defined by

$$
\begin{aligned}
z & \mapsto 0, \\
\operatorname{conv}(i, j) & \mapsto m_{i, j}
\end{aligned}
$$




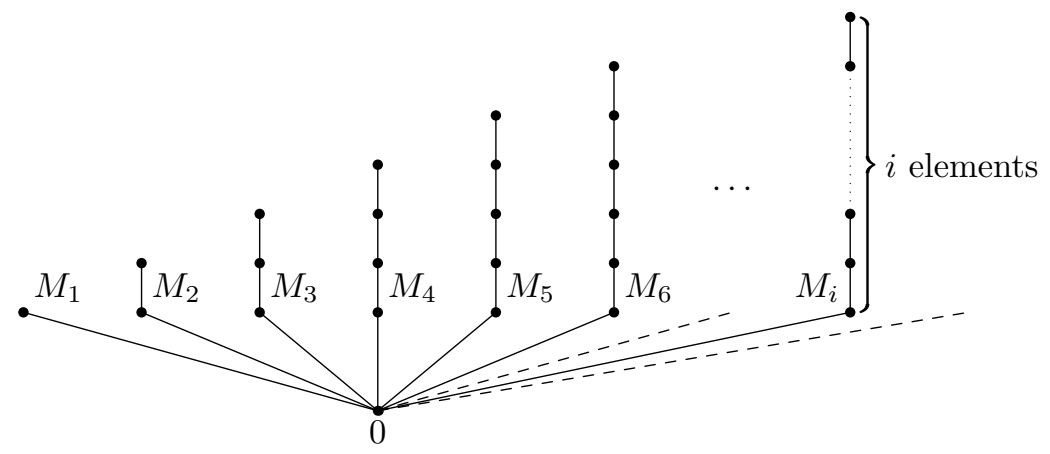

Figure 1: Hasse diagram for $(S, \preceq)$

Notice that the mapping $\phi$ is injective; thus the equality relation is simply

$$
L_{=}=\{(w, w): w \in L\},
$$

and is therefore regular. The order relation is

$$
\begin{aligned}
L_{\preceq} & =\{(z, w): w \in L\} \\
& \cup\{(\operatorname{conv}(i, j), \operatorname{conv}(i, k)): i, j, k \in \mathbb{N}, j \leq k \leq i\} .
\end{aligned}
$$

The relation (5.1) is clearly regular. Moreover, (5.2) is regular since an automaton can check that the first components on each input tape match, that the second components do not exceed the first, and that the second component on the first tape is less than the second component on the second tape.

Furthermore, $(S, \preceq)$ has the following useful property:

Lemma 5.2. Let $(T, \circ)$ be a semigroup containing $(S, \circ)$ as a subsemigroup. Suppose that $(L, \phi)$ is an injective automatic presentation for $(T, \circ)$ and that $J=(S-\{0\}) \phi^{-1}$ is a regular language. Then, for any word $u \in J$ with $u \phi \in M_{i}$ (for some $i \in \mathbb{N}$ ), the set $H_{u}=M_{i} \phi^{-1}$ can be effectively computed, and so the index $i=\left|M_{i}\right|=\left|H_{u}\right|$ can also be computed.

Proof. The first-order formula

$$
\left(u \phi \preceq u_{\max } \phi\right) \wedge(\forall w \in J)\left((u \phi \preceq w \phi) \Longrightarrow\left(w \phi \preceq u_{\max } \phi\right)\right)
$$

is satisfied (in $J$ ) by the unique word $u_{\max }$ such that $u_{\max } \phi$ is the maximum element of $M_{i}$; thus this word $u_{\max }$ can be effectively computed.

Construct the language

$$
H_{u}=\left\{w \in J: w \phi \preceq u_{\max } \phi\right\} ;
$$

then $H_{u}=M_{i} \phi^{-1}$, and so one obtains $i=\left|M_{i}\right|=\left|H_{u}\right|$. 


\section{$6 \quad$ Finite Rees index subsemigroups and extensions}

The Rees index of a subsemigroup $T$ of a semigroup $S$ is defined to be $|S-T|$. If the Rees index of $T$ in $S$ is finite, then $T$ is a large subsemigroup of $S$ and $S$ is a small extension of $T$. Many properties of semigroups are preserved on passing to small extensions and large subsemigroups: for example, finite generation [8], finite presentability [17], and automaticity [13]. Generally, the proofs of these results for passing to small extensions are easy, and the proofs for passing to large subsemigroups are hard. [For example, the proof of the preservation of finite presentability on passing to small extensions [17, Theorem 4.1] is about twenty lines long; the proof for passing to large subsemigroups is over ten pages long [17, p. 388-398] and very technical (a gap in the original proof is fixed in [12]).] In contrast to this is the situation for automatic presentations: the present section exhibits an easy proof of the preservation of FA-presentability on passing to large subsemigroups, and an example to show that FA-presentability is not, in general, closed under passing to small extensions.

Proposition 6.1. Let $S$ be a semigroup with a subsemigroup $T$ of finite Rees index. Then $T$ admits an automatic presentation if $S$ does.

Proof. Let $(L, \phi)$ be an injective automatic presentation for $S$. Let $X$ be the finite set $\{w \in L: w \phi \in S-T\}$. Then $K=L-X$ is regular and $K \phi=T$. Now, $K_{=}=L_{=} \cap(K \times K)$ and $K_{\circ}=L_{\circ} \cap(K \times K)$, so $K_{=}$and $K_{\circ}$ are regular. Thus $\left(K,\left.\phi\right|_{K}\right)$ is an automatic presentation for $T$.

Let $(S, \preceq)$ be the semilattice from Section 5 . Let $Y$ be a non-recursively enumerable subset of $\mathbb{N}$. Let $P$ be the set $S \cup\{e\}$, and extend the relation $\preceq$ to $P$ by defining

$$
\begin{aligned}
0 & \preceq e, \\
m_{i, j} & \preceq e \quad \quad \text { for all } i \in Y \text { and } j \in \mathbb{N} \text { with } j \leq i .
\end{aligned}
$$

The relation is $\preceq$ is a partial order on $P$ and $(P, \preceq)$ is again a semilattice. The Hasse diagram of $(P, \preceq)$ is similar to that of $(S, \preceq)$ : the only difference is that there is a new element $e$ which is above those subchains $M_{i}$ for $i \in Y$; see Figure 2.

Proposition 6.2. The semilattice $(P, \preceq)$ does not admit an automatic presentation.

Proof. Suppose, with the aim of obtaining a contradiction, that $(P, \preceq)$ has an automatic presentation $(L, \phi)$; without loss of generality, suppose that this automatic presentation is injective. Then it is possible to enumerate $Y$ as follows. Let $z \in L$ represent 0 ; let $f \in L$ represent $e$. Let $K=L-\{f, z\}$.

1. Fix an effective enumeration of $K$. 


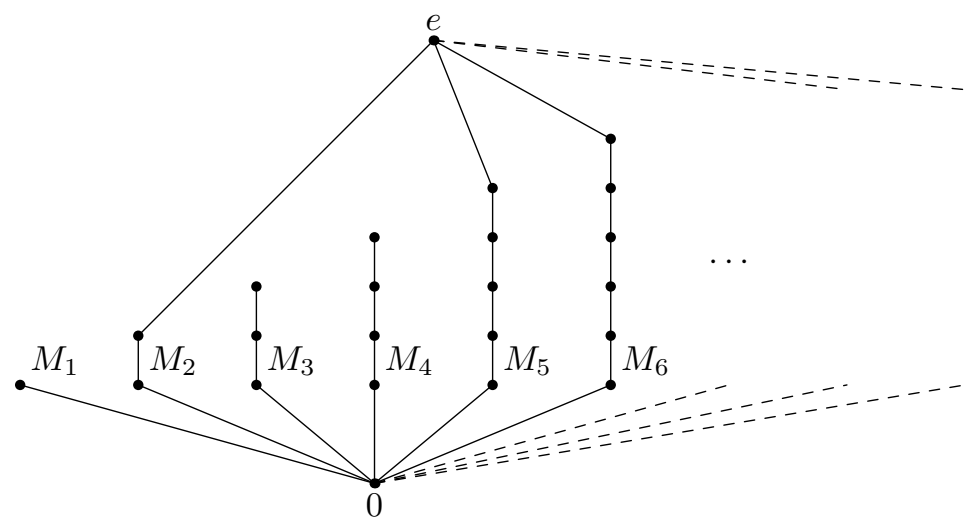

Figure 2: Hasse diagram for $(P, \preceq)$, assuming (for the sake of illustration) that 2,5 , and 6 are in $Y$.

2. For each enumerated word $u \in K$, use Lemma 5.2 to construct $H_{u}$ and so obtain $i=\left|M_{i}\right|=\left|H_{u}\right|$ with $u \phi \in M_{i}$.

3. Now,

$$
i \in Y \Longleftrightarrow(u \phi) \prec(f \phi) .
$$

This first-order condition can be checked: output $i$ if the condition holds.

Now, $(P, \preceq)$ is a finite Rees index extension of $(S, \preceq)$, but the latter is FA-presentable while the former is not. This establishes the following result:

Proposition 6.3. The class of FA-presentable semigroups is not closed under finite Rees index extensions; indeed, it is not closed under extensions of index 1.

However, the class of FA-presentable semigroups is closed under forming two special types of finite Rees index extension: adjoining a zero and adjoining an identity:

Proposition 6.4. Let $S$ be a semigroup. Then:

1. $S$ is FA-presentable if and only if $S^{0}$ is FA-presentable.

2. $S$ is FA-presentable if and only if $S^{1}$ is FA-presentable.

Proof. Suppose $S$ admits an automatic presentation $(L, \phi)$. Let $L^{\prime}=L \cup\{z\}$ where $z$ is a new symbol and extend $\phi$ to $\phi^{\prime}: L^{\prime} \rightarrow S^{0}$ by letting $z \phi=0$. Then $L^{\prime}$ is regular since $L$ is regular and $L^{\prime} \phi=S^{0}$. Furthermore,

$$
L_{\circ}^{\prime}=L_{\circ} \cup\left\{(w, z, z),(z, w, z): w \in L^{\prime}\right\}
$$


is regular since $L_{\circ}$ is regular. So $\left(L^{\prime}, \phi^{\prime}\right)$ is an automatic presentation for $S^{0}$.

If $S^{0}$ admits an automatic presentation, then so does its finite Rees index subsemigroup $S$.

The reasoning for $S^{1}$ is similar.

\section{Finitely generated Clifford semigroups}

The aim of the present section is to characterize those finitely generated Clifford semigroups that admit automatic presentations. Clifford semigroups are a standard notion in semigroup theory, being a species of semigroups that are 'close' to being groups. They admit several equivalent definitions [14, Theorem 4.2.1]. For the purposes of this section, it is best to consider a Clifford semigroup as a particular type of strong semilattice of groups. Recall the definition of the more general concept of a strong semilattice of semigroups:

Definition 7.1. Let $Y$ be a semilattice. For each $\alpha \in Y$, let $S_{\alpha}$ be a semigroup. For $\alpha \geq \beta$, let $\phi_{\alpha, \beta}: S_{\alpha} \rightarrow S_{\beta}$ be a homomorphism such that

1. For each $\alpha \in Y$, the homomorphism $\phi_{\alpha, \alpha}$ is the identity mapping.

2. For all $\alpha, \beta, \gamma \in Y$ with $\alpha \geq \beta \geq \gamma$,

$$
\phi_{\alpha, \beta} \phi_{\beta, \gamma}=\phi_{\alpha, \gamma} .
$$

The strong semilattice of semigroups $S=\mathcal{S}\left[Y ; S_{\alpha} ; \phi_{\alpha, \beta}\right]$ consists of the disjoint union $\bigcup_{\alpha \in Y} S_{\alpha}$ with the following multiplication: if $x \in S_{\alpha}$ and $y \in S_{\beta}$, then

$$
x y=\left(x \phi_{\alpha, \alpha \wedge \beta}\right)\left(y \phi_{\beta, \alpha \wedge \beta}\right),
$$

where $\alpha \wedge \beta$ denotes the greatest lower bound of $\alpha$ and $\beta$.

The definition of a Clifford semigroup is now easy:

Definition 7.2. A Clifford semigroup is a strong semilattice of groups: that is, a semigroup $\mathcal{S}\left[Y ; G_{\alpha} ; \phi_{\alpha, \beta}\right]$, where each $G_{\alpha}$ is a group.

[For further information on Clifford semigroups, see [14, Chapter 4].]

Proposition 7.3. Let $S=\mathcal{S}\left[Y ; G_{\alpha} ; \phi_{\alpha, \beta}\right]$ be an FA-presentable Clifford semigroup. Then the semilattice $Y$ and all the groups $M_{\alpha}$ are FA-presentable.

Proof. Let $(L, \phi)$ be an injective automatic presentation for $S$. Let

$$
E=\left\{w:(w \phi)^{2}=w \phi\right\} ;
$$


this set, being first-order definable, is regular. By definition, $E \phi$ is the set of idempotents in $S$ - the identities of the groups $G_{\alpha}$ - which is isomorphic to the semilattice $Y$. The multiplication in $E \phi$ is simply the restriction of the multiplication in $S$, so the relation

$$
E_{\circ}=L_{\circ} \cap(E \times E)
$$

is regular. So $\left(E,\left.\phi\right|_{E}\right)$ is an automatic presentation for $Y$.

Choose any group $G_{\alpha}$ with identity $1_{\alpha}$. The elements of $G_{\alpha}$ are precisely those elements $x$ of $S$ such that (1) $1_{\alpha}$ acts as an identity on $x$ and (2) there is no idempotent $e$ with $e<1_{\alpha}$ (in the semilattice $E \phi$ ) such that $e$ acts as an identity on $x$. Thus, the subset of $L$ representing elements of $G_{\alpha}$ is precisely

$$
\begin{gathered}
K=\left\{u \in L:(u \phi) 1_{\alpha}=1_{\alpha}(u \phi)=u \phi\right. \\
\wedge(\forall v \in E)\left(\left(v \phi \leq 1_{\alpha}\right) \wedge((u \phi)(v \phi)=(v \phi)(u \phi)=u \phi)\right. \\
\left.\left.\Longrightarrow\left(1_{\alpha}=v \phi\right)\right)\right\} .
\end{gathered}
$$

So $\left(K,\left.\phi\right|_{K}\right)$ is an automatic presentation for $G_{\alpha}$.

Corollary 7.4. Let $S=\mathcal{S}\left[Y ; G_{\alpha} ; \phi_{\alpha, \beta}\right]$ be an FA-presentable finitely generated Clifford semigroup. Then $Y$ is finite and every $G_{\alpha}$ is virtually abelian.

Proof. Since $S$ is finitely generated, the semilattice $Y$, being a homomorphic image of $S$, must also finitely generated. Any finitely generated semilattice is finite; thus $Y$ is finite.

Choose any one of the groups $G_{\alpha}$. By Proposition 7.3, $G_{\alpha}$ is FApresentable. Choose a finite generating set $X$ for $S$, and let $X^{\prime}$ be those elements of $X$ lying in groups $G_{\beta}$ with $\beta \geq \alpha$. Then $G_{\alpha}$ is finitely generated by the images of the elements of $X^{\prime}$ under the various homomorphisms $\phi_{\beta, \alpha}$. Thus, by Theorem $2.8, G_{\alpha}$ is virtually abelian.

Proposition 7.5. Let $G_{1}, \ldots, G_{n}$ be finitely generated virtually abelian groups. Then there is a finite-index normal abelian subgroup $H_{i} \simeq \mathbb{Z}^{n_{i}}$ (for some $n_{i} \in \mathbb{N} \cup\{0\}$ ) of each $G_{i}$ such that, if $\phi: G_{i} \rightarrow G_{j}$ is a homomorphism, then $H_{i} \phi \subseteq H_{j}$.

Proof. Each group $G_{i}$ has an index- $k_{i}$ normal abelian subgroup $J_{i}$ isomorphic to $\mathbb{Z}^{n_{i}^{\prime}}$ for some $n_{i}^{\prime} \in \mathbb{N} \cup\{0\}$. Let $K$ be the least common multiple of the exponents of the various factor groups $G_{i} / J_{i}$. Let $H_{i}$ be the subgroup of $G_{i}$ generated by $\left\{x^{K}: x \in G_{i}\right\}$. By the choice of $K$, every element $x^{K}$ of $G_{i}$ lies in $J_{i}$. So $H_{i}$ is a subgroup of $J_{i}$ and so isomorphic to $\mathbb{Z}^{n_{i}}$ for some $n_{i} \in \mathbb{N} \cup\{0\}$. Furthemore, the factor group $J_{i} / H_{i}$ is a finitely generated abelian group of finite exponent and is therefore finite. So the index of $H_{i}$ in $G_{i}$ is finite. 
Now, let $\phi: G_{i} \rightarrow G_{j}$ be a homomorphism. Any $K$-th power in $G_{i}$ must be mapped to a $K$-th power in $G_{j}$, so the subgroup of $G_{i}$ generated by $K$-th powers - namely $H_{i}$ - must be mapped into the subgroup of $G_{j}$ generated by $K$-th powers - namely $H_{j}$. Finally, each $H_{i}$ is normal in $G_{i}$ since it is generated by all the $K$-th powers.

Proposition 7.6. Let $S=\mathcal{S}\left[Y ; G_{\alpha} ; \phi_{\alpha, \beta}\right]$ be a finitely generated Clifford semigroup where each group $G_{\alpha}$ is virtually abelian. Then $S$ admits an automatic presentation.

Proof. Since $S$ is finitely generated, the semilattice $Y$ must be finite.

By Proposition 7.5, one can view each group $G_{\alpha}$ as having a finite-index normal abelian subgroup $H_{\alpha} \simeq \mathbb{Z}^{n_{\alpha}}$ such that for all $\alpha, \beta \in Y$ with $\alpha \geq \beta$, $H_{\alpha} \phi_{\alpha, \beta} \subseteq H_{\beta}$.

For each $\alpha \in Y$, choose a set of representatives $g_{\alpha, 1}, \ldots, g_{\alpha, l_{\alpha}}$ for each of the cosets of $H_{\alpha}$ in $G_{\alpha}$. Define the language

$$
L_{\alpha}=\left\{g_{\alpha, i} \operatorname{conv}\left(\epsilon_{1} z_{1}, \ldots, \epsilon_{n} z_{n_{\alpha}}\right): i=1, \ldots, l_{\alpha}, \epsilon_{i} \in\{+,-\}, z_{j} \in \mathbb{N} \cup\{0\}\right\},
$$

where the various $z_{i}$ are understood to be in reverse binary notation. The language $L_{\alpha}$, being a finite union of regular languages, is itself regular. The virtually abelian group $G_{\alpha}$ admits $L_{\alpha}$ as an automatic presentation. Let $L=\bigcup_{\alpha \in Y} L_{\alpha}$. The aim is to show that $L$ is an automatic presentation for $S$.

The first task is to show that the homomorphisms $\phi_{\alpha, \beta}$ are all regular (that is, the relations $\phi_{\alpha, \beta}$ are regular as relations). Now, $\phi_{\alpha, \beta}$ is determined by the images of the various $g_{\alpha, i}$ and the elements $(1,0, \ldots, 0),(0,1, \ldots, 0)$, $\ldots,(0, \ldots, 0,1)$. Suppose that, for $i=1, \ldots, l_{\alpha}$,

$$
g_{\alpha, i} \phi_{\alpha, \beta}=g_{\beta, j_{i}}\left(z_{\beta, i, 1}, \ldots, z_{\beta, i, n_{\beta}}\right)
$$

and

$$
\underbrace{(\ldots, 0,1,0, \ldots)}_{i \text {-th coordinate is } 1} \phi_{\alpha, \beta}=\left(y_{\beta, i, 1}, \ldots, y_{\beta, i, n_{\beta}}\right) .
$$

(Recall that $H_{\alpha} \phi_{\alpha, \beta} \subseteq H_{\beta}$.)

So

$$
\begin{aligned}
& \left(g_{\alpha, i}\left(z_{1}, \ldots, z_{n_{\alpha}}\right)\right) \phi_{\alpha, \beta} \\
& \quad=g_{\beta, j_{i}}\left(\left(z_{\beta, i, 1}, \ldots, z_{\beta, i, n_{\beta}}\right)+\sum_{j=1}^{n_{\alpha}} z_{j}\left(y_{\beta, j, 1}, \ldots, y_{\beta, j, n_{\beta}}\right)\right) .
\end{aligned}
$$


That is,

$$
\begin{gathered}
\phi_{\alpha, \beta}=\left\{\left(g_{\alpha, i}\left(z_{1}, \ldots, z_{n_{\alpha}}\right), g_{\beta, j_{i}}\left(x_{1}, \ldots, x_{n_{\beta}}\right)\right): i \in\left\{1, \ldots, l_{\alpha}\right\},\right. \\
(\mathbb{Z},+) \models \\
\left(x_{1}=z_{\beta, i, 1}+\sum_{j=1}^{n_{\alpha}} z_{j} y_{\beta, j, 1}\right) \wedge \\
\left(x_{2}=z_{\beta, i, 2}+\sum_{j=1}^{n_{\alpha}} z_{j} y_{\beta, j, 2}\right) \wedge \\
\vdots \\
\left.\left(x_{n_{\beta}}=z_{\beta, i, n_{\beta}}+\sum_{j=1}^{n_{\alpha}} z_{j} y_{\beta, j, n_{\beta}}\right)\right\},
\end{gathered}
$$

where $z_{j} y_{\beta, j, h}$ is understood to be an abbreviation for

$$
\underbrace{z_{j}+\ldots+z_{j}}_{y_{\beta, j, h} \text { times }} .
$$

By Proposition 2.7, the relation $\phi_{\alpha, \beta}$ is regular.

Define

$$
\begin{aligned}
M_{\alpha, \beta}=\{(u, v, w): & u \in L_{\alpha}, v \in L_{\beta}, w \in L_{\alpha \wedge \beta}, \\
& \left(\exists u^{\prime}, v^{\prime} \in L_{\alpha \wedge \beta}\right)\left(\left(u, u^{\prime}\right) \in \phi_{\alpha, \alpha \wedge \beta},\right. \\
& \left.\left.\left(v, v^{\prime}\right) \in \phi_{\beta, \alpha \wedge \beta},\left(u^{\prime}, v^{\prime}, w\right) \in\left(L_{\alpha \wedge \beta}\right)_{\circ}\right)\right\} .
\end{aligned}
$$

This language is regular and describes the multiplication of elements in $G_{\alpha}$ and $G_{\beta}$. Finally, let

$$
M=\bigcup_{\alpha, \beta \in Y} M_{\alpha, \beta} .
$$

By the definition of a Clifford semigroup, the regular relation $M$ describes multiplication in $S$. This completes the proof.

From Corollary 7.4 and Proposition 7.6 follows immediately the characterization of those finitely generated Clifford semigroups admitting automatic presentations:

Theorem 7.7. Let $S=\mathcal{S}\left[Y ; G_{\alpha} ; \phi_{\alpha, \beta}\right]$ be a finitely generated Clifford semigroup. Then $S$ admits an automatic presentation if and only if each group $G_{\alpha}$ is virtually abelian.

In light of Theorem 7.7, one naturally asks whether the class of FApresentable semigroups is closed under forming strong semilattices of semigroups. The following counterexample shows that this generalization does not obtain, every when the semilattice is finite: 


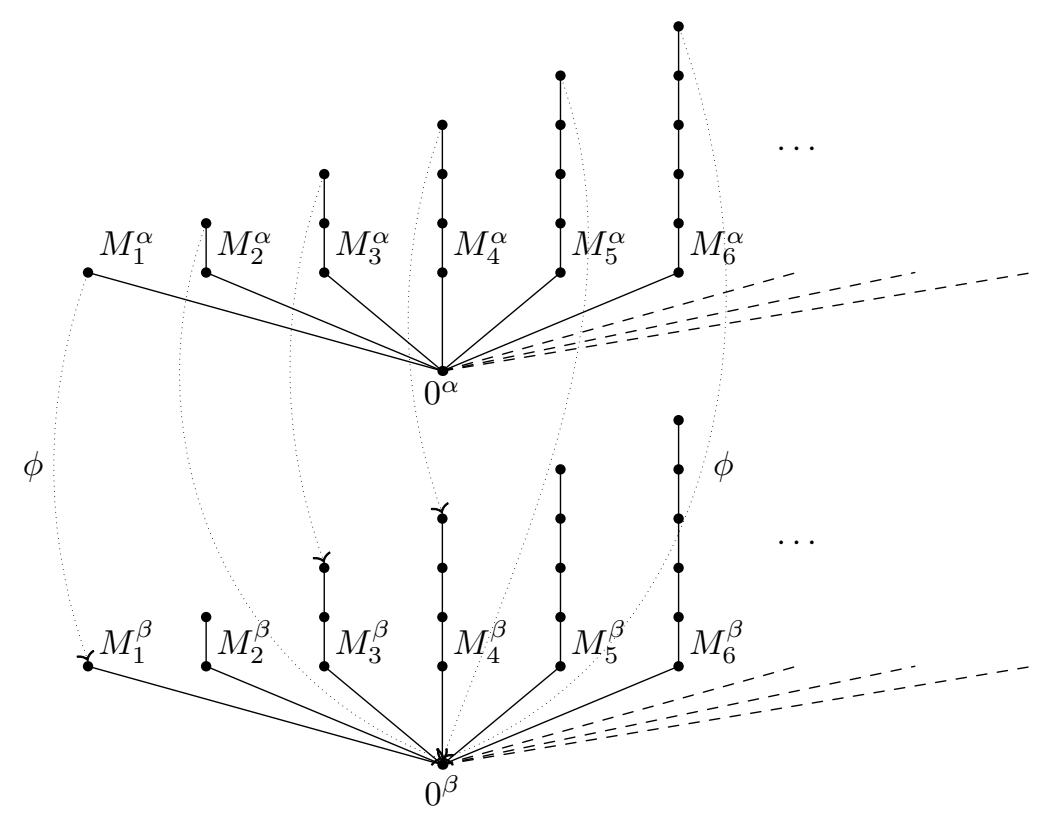

Figure 3: Schematic diagram of the semigroup $T=\mathcal{S}\left[Y ; S_{\alpha} ; \phi_{\alpha, \beta}\right]$, assuming (for the sake of illustration) that 2,5 , and 6 are in $X$. The dotted lines indicate the mapping $\phi_{\alpha, \beta}$.

Example 7.8. Let $Y=\{\alpha, \beta\}$ be the two-element semilattice with $\beta<\alpha$. Let $S_{\alpha}$ and $S_{\beta}$ be two copies of the semilattice from Section 5. (Distinguish elements and subsets of these two semilattices using superscript symbols $\alpha$ or $\beta$, as appropriate.) Let $X$ be a non-recursively enumerable subset of the natural numbers. Define the mapping $\phi_{\alpha, \beta}$ by

$$
\begin{aligned}
& 0^{\alpha} \mapsto 0^{\beta} \\
& m_{i, j}^{\alpha} \mapsto\left\{\begin{array}{ll}
0^{\beta} & \text { if } i \in X \\
m_{i, j}^{\beta} & \text { if } i \notin X
\end{array}\right\} \text { for } i, j \in \mathbb{N} \text { with } j \leq i .
\end{aligned}
$$

The mapping $\phi_{\alpha, \beta}$ is a homomorphism. Let $T$ be the strong semilattice of semigroups $\mathcal{S}\left[Y ; S_{\alpha} ; \phi_{\alpha, \beta}\right]$; see Figure 3 for a schematic diagram of $T$.

For reductio ad absurdum, suppose that $T$ admits an automatic presentation $(L, \phi)$. Without loss of generality, assume $(L, \phi)$ is injective. Let $y \in L$ be such that $y \phi=0^{\alpha}$. Let

$$
K^{\prime}=\{w \in L:(w \phi)(y \phi)=(y \phi)\} ;
$$

then $K^{\prime}$ is regular and $K^{\prime}=S_{\alpha} \phi^{-1}$. Let $J=L-K^{\prime}$; then $J=S_{\beta} \phi^{-1}$. Let $z \in J$ be such that $z \phi=0^{\beta}$. Let $K=K^{\prime}-\{y\}$. So $J$ and $K$ are both regular.

The contradiction arises from the following procedure, which enumerates the non-recursively enumerable set $X$ : 
1. Fix an effective enumeration of $K$.

2. For each enumerated word $u \in K$, use Lemma 5.2 to construct $H_{u}$ and so obtain $i=\left|M_{i}\right|=\left|H_{u}\right|$ with $u \phi \in M_{i}$.

3. Now,

$$
i \in X \Longleftrightarrow(u \phi) \phi_{\alpha, \beta}=0^{\beta} \Longleftrightarrow(\forall v \in J)((v \phi)(u \phi)=(z \phi)) .
$$

This first-order condition can be checked: output $i$ if the condition holds.

\section{Completely simple \& completely 0-simple semi- groups}

Definition 8.1. Let $S$ be a semigroup, $I$ and $\Lambda$ be index sets, and $P$ be a $\Lambda \times I$ matrix over $S \cup\{0\}$ whose $\lambda, i$-th element is $p_{\lambda, i}$. The Rees matrix semigroup $\mathcal{M}^{0}[S ; I, \Lambda ; P]$ is defined to be the set $(I \times S \times \Lambda) \cup\{0\}$ with multiplication

$$
(i, g, \lambda)(j, h, \mu)= \begin{cases}\left(i, g p_{\lambda, j} h, \mu\right) & \text { if } p_{\lambda, j} \neq 0 \\ 0 & \text { if } p_{\lambda, j}=0\end{cases}
$$

and

$$
(i, g, \lambda) 0=0(i, g, \lambda)=00=0 .
$$

Now restrict $P$ to be a matrix over $S$. The Rees matrix semigroup $\mathcal{M}[S ; I, \Lambda ; P]$ is defined to be the set $I \times S \times \Lambda$ with multiplication

$$
(i, g, \lambda)(j, h, \mu)=\left(i, g p_{\lambda, j} h, \mu\right) .
$$

Recall that a semigroup is completely 0 -simple if it has no proper twosided ideals, is not the two-element null semigroup, and contains a primitive idempotent (that is, an idempotent $e$ such that, for all idempotents $f$, $e f=f e=f \Longrightarrow e=f)$. The celebrated Rees theorem [14, Section 3.2] asserts that all completely 0 -simple semigroups are isomorphic to a semigroup $\mathcal{M}^{0}[G ; I, \Lambda ; P]$, where $G$ is a group and $I$ and $\Lambda$ are finite sets.

Proposition 8.2. Let $G$ be a group. If the completely 0-simple semigroup $\mathcal{M}^{0}[G ; I, \Lambda ; P]$ is FA-presentable, then the group $G$ is FA-presentable.

Proof. Choose a word $w \in L$ such that $w \phi$ is idempotent. Then the $\mathcal{H}$-class containing $w \phi$ is manifestly non-null and so isomorphic to $G$ [14, Proposition 2.3.6]. Let $Q$ be the subset of $L$ consisting of words representing elements that are $\mathcal{H}$-related to $w \phi$; since Green's relations are first-order definable, the language $Q$ is regular. Therefore $\left(Q,\left.\phi\right|_{Q}\right)$ is an automatic presentation for the $\mathcal{H}$-class containing $w \phi$, which is isomorphic to $G$. So $G$ is FA-presentable. 
Corollary 8.3. Let $G$ be a group. If the completely simple semigroup $\mathcal{M}[G ; I, \Lambda ; P]$ is FA-presentable, then the group $G$ is FA-presentable.

Proof. Let $S=\mathcal{M}[G ; I, \Lambda ; P]$. Then $S^{0} \simeq \mathcal{M}^{0}[G ; I, \Lambda ; P]$. Now, since $S$ is FA-presentable, then $S^{0}$ is FA-presentable (by Proposition 6.4), and so $G$ is FA-presentable (by Proposition 8.2).

Corollary 8.3 no longer holds if one generalizes to Rees matrix semigroups over arbitrary base semigroups. To see this, let $X$ be a finite alphabet and let $F$ be the free semigroup over $X$. By Theorem 2.9, the semigroup $F$ is not FA-presentable, since it does not have polynomial growth. Let $T=\mathcal{M}\left[F^{0}, I, \Lambda, P\right]$, where $I$ and $\Lambda$ are arbitrary finite sets and every entry of the matrix $P$ is 0 . Every product in $T$ is therefore an element of the form $(i, 0, \lambda)$. Let

$$
L=\left\{i \lambda w: i \in I, \lambda \in \Lambda, w \in F^{0}\right\}
$$

(allowing a slight abuse of notation in viewing $F^{0}$ as both a semigroup and a set of words) and define $\phi: L \rightarrow T$ by $(i \lambda w) \phi=(i, w, \lambda)$. Then $(L, \phi)$ is an automatic presentation for $T$, since

$$
L_{=}=\{(u, u): i \in L\}
$$

and

$$
\begin{aligned}
L_{\circ} & =\{(i \lambda u, j \mu v, i \mu 0): i, j \in I, \lambda, \mu \in \Lambda, u, v \in F\} \\
& =\{(i \lambda, j \mu, i \mu)\}\{(u, v, 0): u, v \in F\},
\end{aligned}
$$

which is regular since it is the concatenation of a finite relation and one that is manifestly regular. So $T$ is FA-presentable, but the underlying semigroup $F^{0}$ is not.

Proposition 8.4 ([1]). The completely 0 -simple semigroup $\mathcal{M}^{0}[G ; I, \Lambda ; P]$ is finitely generated if and only if the group $G$ is finitely generated and the sets $I$ and $\Lambda$ are finite.

Proposition 8.5. Let $G$ be FA-presentable and let $I$ and $\Lambda$ be finite. Then, for any $\Lambda \times I$ matrix $P$ over $G^{0}$, the Rees matrix semigroup $\mathcal{M}^{0}[G ; I, \Lambda ; P]$ is FA-presentable.

Proof. Let $(L, \phi)$ be an automatic presentation for $G$. Let

$$
K=\{i \lambda w: i \in I, \lambda \in \Lambda, w \in L\} \cup\{z\},
$$

and let $\psi: K \rightarrow \mathcal{M}^{0}[G ; I, \Lambda ; P]$ be defined by

$$
i \lambda w \mapsto(i, w \phi, \lambda), \quad z \mapsto 0 .
$$


The aim is now to show that $(K, \psi)$ is an automatic presentation for the semigroup $\mathcal{M}^{0}[G ; I, \Lambda ; P]$. The equality relation is

$$
K_{=}=\left\{(i \lambda u, i \lambda v): i \in I, \lambda \in \Lambda,(u, v) \in L_{=}\right\} \cup\{(z, z)\},
$$

which is regular since $L_{=}$is regular. Now fix $\lambda \in \Lambda$ and $j \in I$ and observe that the relation

$$
\begin{gathered}
K_{\circ}^{\lambda, j}=\left\{(i \lambda u, j \mu v, i \mu w): i \in I, \mu \in \Lambda, u, v, w \in L,(u \phi) p_{\lambda, j}(v \phi)=w \phi\right\} \\
\cup\left\{(i \lambda u, j \mu v, z): i \in I, \mu \in \Lambda, u, v \in L, p_{\lambda, j}=0\right\},
\end{gathered}
$$

being first-order definable in terms of $L_{\circ}$, is regular. So the relation

$$
K_{\circ}=\{(z, z, z),(z, i \lambda u, z),(i \lambda u, z, z): u \in L\} \cup \bigcup_{\substack{\lambda \in \Lambda \\ j \in I}} K_{\circ}^{\lambda, j}
$$

is regular.

Theorem 8.6. A finitely generated completely 0 -simple semigroup $\mathcal{M}^{0}[G ; I, \Lambda ; P]$ is FA-presentable if and only if the group $G$ is virtually abelian.

Proof. Let $S=\mathcal{M}^{0}[G ; I, \Lambda ; P]$.

Suppose $S$ is FA-presentable. Then by Proposition 8.2, the group $G$ is FA-presentable. The semigroup $S$ is finitely generated, and so, by Proposition 8.4, the group $G$ is also finitely generated. Therefore, by Theorem 2.8, $G$ is virtually abelian.

For the converse, suppose $G$ is virtually abelian. Since $S$ is finitely generated, $G$ is also, and so by Theorem 2.8, $G$ is FA-presentable. Thus, by Proposition 8.5, the semigroup $S$ is FA-presentable.

Corollary 8.7. A finitely generated completely simple semigroup $\mathcal{M}[G ; I, \Lambda ; P]$ has an automatic presentation if and only if the group $G$ is virtually abelian.

Proof. Let $S=\mathcal{M}[G ; I, \Lambda ; P]$. Then $S^{0}=\mathcal{M}^{0}[G ; I, \Lambda ; P]$. Now, $S$ admits an automatic presentation if and only if $S^{0}$ admits an automatic presentation (by Proposition 6.4) if and only if $G$ is virtually abelian.

Corollary 8.7 parallels an established result for automatic completely simple semigroups: if $S$ is a finitely generated completely simple semigroup $\mathcal{M}[G ; I, J ; P]$, then $\mathrm{S}$ is automatic if and only if the group $G$ is automatic $[10]$. 


\section{Bruck-Reilly extensions}

This section shows that if a Bruck-Reilly extension admits an automatic presentation, then so does its base semigroup (Proposition 9.2). An example is then exhibited to show that a Bruck-Reilly extension of an FA-presentable semigroup need not be FA-presentable (Example 9.3). This parallels the situation for automatic (in the sense of $[11,9]$ ) semigroups: the first author proved that an automatic Bruck-Reilly extension has an automatic underlying semigroup [5, Theorem 3.3], and that the class of automatic semigroups is not closed under forming Bruck-Reilly extensions [5, Theorem 5.2].

Definition 9.1. Let $S$ be a monoid and let $\theta$ be an endomorphism of $S$. The Bruck-Reilly extension $\operatorname{BR}(S, \theta)$ is the semigroup

$$
(\mathbb{N} \cup\{0\}) \times S^{I} \times(\mathbb{N} \cup\{0\})
$$

(where $S^{I}$ is $S$ with an identity adjoined unless one is already present), with the multiplication

$$
(m, s, n)(p, t, q)= \begin{cases}\left(m, s\left(t \theta^{n-p}\right), q+n-p\right) & \text { if } n \geq p \\ \left(m+p-n,\left(s \theta^{p-n}\right) t, q\right) & \text { if } n \leq p .\end{cases}
$$

If $S$ is presented by $\langle A \mid \mathcal{R}\rangle$, then the Bruck-Reilly extension $\operatorname{BR}(S, \theta)$ is presented by

$$
\langle A \cup\{b, c\} \mid \mathcal{R},(b c, 1),(b a,(a \theta) b),(a c, c(a \theta)): a \in A\rangle .
$$

Every element of $\operatorname{BR}(S, \theta)$ can be expressed as a word of the form $c^{\gamma} w b^{\beta}$ where $w \in A^{*}$ and $\gamma, \beta \in \mathbb{N} \cup\{0\}$. Furthermore, the exponents $\gamma$ and $\beta$ are uniquely determined.

Proposition 9.2. Let $S$ be a semigroup and $\theta$ an endomorphism of $S$. Suppose $\operatorname{BR}(S, \theta)$ admits an automatic presentation. Then $S$ admits an automatic presentation.

Proof. Let $(L, \phi)$ be an automatic presentation for $\operatorname{BR}(S, \theta)$. Let $u, v \in L$ be such that $u \phi=b$ and $v \phi=c$. (The elements $b$ and $c$ are as in (9.1).) Let $D$ be the set of words in $L$ representing right multiples of $c$. Since

$$
D=\left\{w \in L:(\exists p \in L)\left((v, p, w) \in L_{\circ}\right)\right\},
$$

the set $D$ is regular. Similarly, the set $G$ of words representing left multiples of $b$ is regular. The elements of $S$ are precisely those elements of $\operatorname{BR}(S, \theta)$ that are neither right multiples of $c$ nor left multiples of $b$. So $(L-(D \cup$ $G)) \phi=S$. Therefore $\left(L-(D \cup G),\left.\phi\right|_{L-(D \cup G)}\right)$ is an automatic presentation for $S$. So $S$ too admits an automatic presentation. 


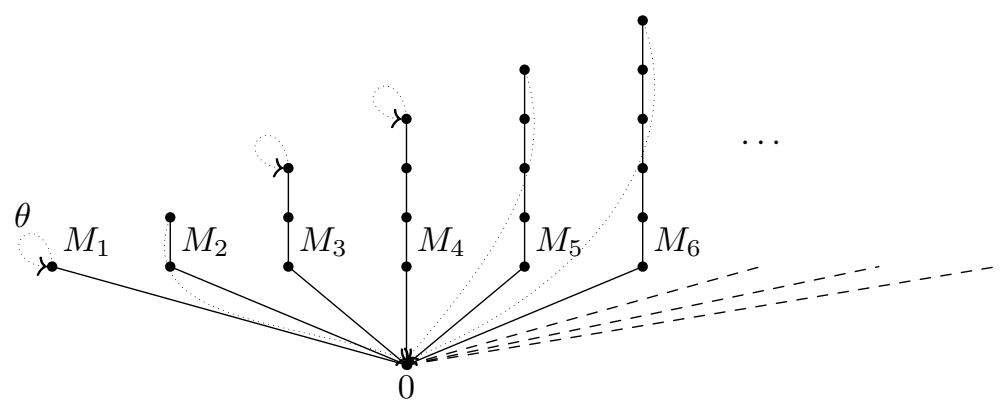

Figure 4: Schematic diagram of the Bruck-Reilly extension $\operatorname{BR}(S, \theta)$, assuming (for the sake of illustration) that 2,5 , and 6 are in $Y$.

Example 9.3. Let $(S, \preceq)$ be the semilattice from Section 5 . Let $Y$ be a non-recursively enumerable subset of the natural numbers. Define $\theta: S \rightarrow S$ by

$$
\begin{aligned}
0 & \mapsto 0 \\
m_{i, j} & \mapsto\left\{\begin{array}{ll}
0 & \text { if } i \in Y \\
m_{i, j} & \text { if } i \notin Y
\end{array}\right\} \text { for } i, j \in \mathbb{N} \text { with } j \leq i .
\end{aligned}
$$

It is easy to see that $\theta$ is an endomorphism of the semilattice $(S, \preceq)$.

Suppose $\operatorname{BR}(S, \theta)$ admits an automatic presentation $(L, \phi)$; without loss of generality, suppose that this automatic presentation is injective. Then it is possible to enumerate $Y$ as follows. Construct the subset $K^{\prime}$ of $L$ with $K^{\prime} \phi=S$; by the proof of the preceding result, $K^{\prime}$ is regular. Let $z \in K^{\prime}$ represent 0 ; let $K=K^{\prime}-\{z\}$

1. Fix an effective enumeration of the regular language $K$.

2. For each enumerated word $u \in K$, use Lemma 5.2 to construct $H_{u}$ and so obtain $i=\left|M_{i}\right|=\left|H_{u}\right|$ with $u \phi \in M_{i}$.

3. Now,

$$
i \in Y \Longleftrightarrow(u \phi) \theta=0 \Longleftrightarrow b(u \phi) c=0 .
$$

(The elements $b$ and $c$ are as in (9.1).) This first-order condition can be checked: output $i$ if the condition holds.

This procedure enumerates $Y$. This is a contradiction, so $\operatorname{BR}(S, \theta)$ does not admit an automatic presentation.

\section{Zero-direct unions}

For each $i \in I$, let $S_{i}$ be a semigroup with a zero $0_{i}$. Their zero-direct union is the semigroup formed by taking their disjoint union, identifying 
their zeroes, and defining the product $s \in S_{i}$ and $t \in S_{j}$ to be this zero if $i \neq j$, or $s t \in S_{i}$ if $i=j$.

The class of FA-presentable semigroups is closed under forming finite zero-direct unions:

Proposition 10.1. Let $S$ and $T$ be semigroups with zeroes and suppose both are FA-presentable. Then their zero-direct union also admits an automatic presentation.

Proof. Let $(L, \phi)$ and $(M, \psi)$ be injective automatic presentations for $S$ and $T$ respectively, with $L$ and $M$ being disjoint. Let $x \in L$ and $y \in M$ be the representatives of the zeroes of $S$ and $T$ respectively. Let $K=L \cup M$. Define $\theta$ from $K$ to the zero-direct union of $S$ and $T$ by $w \theta=w \phi$ for $w \in L$ and $w \theta=w \psi$ for $w \in M$. To see that $(K, \theta)$ is an automatic presentation for the zero-direct union of $S$ and $T$, reason as follows: the equality relation is

$$
K_{=}=\{(x, y),(y, x)\} \cup\{(w, w): w \in K\},
$$

which is manifestly regular, and the multiplication relation is

$$
\begin{aligned}
K_{\circ}= & L_{\circ} \cup M_{\circ} \\
& \cup\left\{(u, v, y):(u, v, x) \in L_{\circ}\right\} \\
& \cup\left\{(u, v, x):(u, v, y) \in M_{\circ}\right\} \\
& \cup\{(u, v, x),(u, v, y),(v, u, x),(v, u, y): u \in L, v \in M\},
\end{aligned}
$$

which is easily seen to be regular since $L_{\circ}, M_{\circ}, L$, and $M$ are all regular.

However, the converse does not hold: the FA-presentability of a zerodirect union does not imply the FA-presentability of the original semigroups:

Example 10.2. Let $S$ be the semilattice from Section 5. Let $Y$ be a nonrecursively enumerable subset of the natural numbers. Let

$$
T=\{z\} \cup \bigcup_{i \in Y} M_{i} \text { and } U=\{z\} \cup \bigcup_{i \in \mathbb{N}-Y} M_{i} .
$$

Then $S$ is the zero-direct union of $T$ and $U$, each of which is the zero-direct union of countably many subsemilattices $M_{i} \cup\{z\}$ (each of which is finite and thus FA-presentable).

Suppose $T$ admits an injective automatic presentation $(L, \phi)$. Then it is possible to enumerate $Y$ as follows. Let $z \in L$ represent the zero of $T$. Let $K=L-\{z\}$.

As in the proof of Proposition 5.2, fix an effective enumeration of $K$. For each enumerated word $w \in K$ (lying in some $M_{i}$ ), construct $H \subseteq L$ with $H \phi=M_{i}$. The language $H$ is finite and its cardinality is $i$; output this $i$. Now replace $K$ by $K-H$ and repeat. This procedure enumerates $Y$. This is a contradiction, so $T$ does not admit an automatic presentation. 
Thus $T$ is a non-FA-presentable semigroup that forms a component of an FA-presentable zero-direct union $S$.

In the preceding example, $T$ is a non-FA-presentable countable zerodirect union of FA-presentable semigroups $M_{i} \cup\{z\}$. Thus the class of FA-presentable semigroups is not closed under countable zero-direct unions. However, it is closed under countable zero-direct unions of isomorphic semigroups:

Proposition 10.3. Let $S$ be a semigroup with a zero that admits an automatic presentation. Let $S_{i}$ be isomorphic to $S$ for all $i \in \mathbb{N}$. Then the zero-direct union of the $S_{i}$ admits an automatic presentation.

Proof. Let $T$ be the zero-direct union of the $S_{i}$. Let $(L, \phi)$ be an injective automatic presentation for $S$; let $y \in L$ be such that $y \phi$ is the zero of $S$. Let $K$ be the language

$$
\{\operatorname{conv}(u, i): u \in L, i \in \mathbb{N}\},
$$

where $i$ is in reverse binary notation. Observe that $K$ is regular. Define $\psi: K \rightarrow T$ by letting $(\operatorname{conv}(u, i)) \psi$ be the element $u \phi$ lying in $S_{i}$. Then

$$
\begin{aligned}
K_{=} & =\{(\operatorname{conv}(y, i), \operatorname{conv}(y, j)): i, j \in \mathbb{N}\} \\
& \cup\{(w, w): w \in K\}
\end{aligned}
$$

and

$$
\begin{aligned}
K_{\circ} & =\left\{(\operatorname{conv}(u, i), \operatorname{conv}(v, i), \operatorname{conv}(w, i)):(u, v, w) \in L_{\circ}, u, v, w \in L-\{y\}, i \in \mathbb{N}\right\} \\
& \cup\left\{(\operatorname{conv}(u, i), \operatorname{conv}(v, i), \operatorname{conv}(y, j)):(u, v, y) \in L_{\circ}, i, j \in \mathbb{N}\right\} \\
& \cup\{(\operatorname{conv}(u, i), \operatorname{conv}(v, j), \operatorname{conv}(y, k)): i, j, k \in \mathbb{N}, i \neq j\},
\end{aligned}
$$

both of which are easily seen to be regular since $L_{\circ}$ is regular and the equality of natural numbers in reverse binary notation can be checked by a finite state automaton.

\section{Semidirect and wreath products}

Let $S$ and $T$ be monoids. Denote by $S^{\oplus T}$ the set of functions from $T$ to $S$ with finite support (that is, where only finitely many elements of $T$ have non-identity image in $S$ ). The [restricted] wreath product $S \_T$ of monoids $S$ and $T$ is the set $S^{\oplus T} \times T$ under the operation

$$
(f, t)\left(g, t^{\prime}\right)=\left(f g^{t}, t t^{\prime}\right), \text { for } f, g: T \rightarrow S \text { and } t, t^{\prime} \in T,
$$

where $g^{t}: T \rightarrow S$ is defined by $(x) g^{t}=(x t) g$ and $f g^{t}: T \rightarrow S$ by $(x) f g^{t}=$ $((x) f)\left((x) g^{t}\right)$. 
Proposition 11.1. Let $S$ be FA-presentable and let $T$ be finite. Then $S \nmid T$ is FA-presentable.

Proof. Suppose $T=\left\{t_{1}, \ldots, t_{k}\right\}$. Let $(L, \phi)$ be an injective automatic presentation for $S$. Let

$$
M=T\left\{\operatorname{conv}\left(u_{1}, \ldots, u_{k}\right): u_{i} \in L\right\},
$$

where $T$ is treated as an abstract set of symbols. Observe that $M$ is a concatenation of a finite language and a regular language and is thus itself regular. Define $\psi: M \rightarrow S \imath T$ by

$$
t \operatorname{conv}\left(u_{1}, \ldots, u_{k}\right) \mapsto(f, t),
$$

where $f: T \rightarrow S$ is defined by $t_{i} \mapsto u_{i} \phi$.

To see that $(M, \psi)$ is an automatic presentation for $S \imath T$, note first that every element of $S \imath T$ has a unique representative in $M$ (since $\phi$ is injective). Now consider the automaton reading a triple

$$
\left(t \operatorname{conv}\left(u_{1} \cdots u_{k}\right), t^{\prime} \operatorname{conv}\left(v_{1}, \ldots, v_{k}\right), s \operatorname{conv}\left(w_{1}, \ldots, w_{k}\right)\right)
$$

to check whether it lies in $M_{\circ}$. First of all, the automaton checks that $s=t t^{\prime}$. Then, for each $i \in\{1, \ldots, k\}$, it finds $j$ such that $t_{j} t=t_{i}$ and checks that $\left(u_{i}, v_{j}, w_{i}\right) \in L_{\circ}$. (That this is possible inside a finite automaton is due to the finiteness of $T$.)

On the other hand, if $S$ is finite and $T$ FA-presentable, then $S \imath T$ need not be FA-presentable. For example, let $S=\{1, c\}$ be the cyclic group of order 2. Define mappings $f, g: \mathbb{Z} \rightarrow S$ by $z f=1$ for all $z \in \mathbb{Z}$ and by $0 g=c$ and $z g=1$ for $z \in \mathbb{Z}-\{0\}$. Then the subsemigroup of $S \imath \mathbb{Z}$ generated by $(f, 1)$ and $(g, 1)$ is free, and so, by Theorem $2.9, S \geq \mathbb{Z}$ cannot be FA-presentable.

The following example shows that class of FA-presentable semigroups is not closed under forming semidirect products, even if the top semigroup is finite.

Example 11.2. Let $(S, \preceq)$ be the semilattice from Section 5 and let $\theta$ : $S \rightarrow S$ be the endomorphism defined using a non-recursively enumerable subset $Y$ of $\mathbb{N}$ in Example 9.3. Let $X=\{$ id, $\theta\}$, where id is the identity mapping on $S$. Then $X$ is a subsemigroup of End $S$, since $\theta^{2}=\theta$. Let $T$ be the semidirect product $X \ltimes S$, where $X$ acts on $S$ (from the right) in the obvious manner. Identify $S$ with the subsemigroup of $T$ consisting of elements of the form [id, $s]$ for some $s \in S$.

Suppose $T$ admits an injective automatic presentation $(L, \phi)$. Let $z, z^{\prime} \in$ $L$ be such that $z \phi=[\mathrm{id}, 0]$ and $z^{\prime} \phi=[\theta, 0]$. Let

$$
K^{\prime}=\{w \in L:(w \phi)(z \phi)=(z \phi)\} ;
$$

then $K^{\prime} \phi=S$. Let $K=K^{\prime}-\{z\}$. Let $J=L-K^{\prime}$. Observe that $K$ and $J$ are regular languages. 
1. Fix an effective enumeration of the regular language $K$.

2. For each enumerated word $u \in K$, use Lemma 5.2 to construct $H_{u}$ and so obtain $i=\left|M_{i}\right|=\left|H_{u}\right|$ with $u \phi \in M_{i}$.

3. Now,

$$
i \in Y \Longleftrightarrow(u \phi) \theta=(z \phi) \Longleftrightarrow(\forall v \in J)((u \phi)(v \phi)=(z \phi)) .
$$

This first-order condition can be checked: output $i$ if the condition holds.

This procedure enumerates $Y$. This is a contradiction, so $T$ does not admit an automatic presentation.

[The semidirect product defined in the preceding example is actually isomorphic to the strong semilattice of semigroups defined in Example 7.8.]

Since a finitely generated group is FA-presentable if and only if it is virtually abelian, the class of finitely generated FA-presentable groups is closed under semidirect products with finite groups. It remains open whether the class of all FA-presentable groups is closed under semidirect products with finite groups, or under finite extensions generally.

\section{Ideals \& quotients}

Let $S$ be a semigroup with an automatic presentation $(L, \phi)$. Let $K$ be a regular (possibly finite) subset of $L$. Then the ideal generated by $K \phi$ is the set of elements represented by the regular language

$$
\begin{aligned}
I(K)=\{w \in L & :(\exists p, q \in L)(\exists u \in K)((p \phi)(u \phi)(q \phi)=(w \phi)) \\
& \vee(\exists p \in L)(\exists u \in K)((p \phi)(u \phi)=(w \phi)) \\
& \vee(\exists q \in L)(\exists u \in K)((u \phi)(q \phi)=(w \phi)) \\
& \vee(\exists u \in K)((u \phi)=(w \phi))\} .
\end{aligned}
$$

One can therefore test membership of ideals generated by subsets described by regular languages. Furthermore, one can test whether such ideals are principal: simply check whether the regular language

$$
\{v \in L:(\forall w \in K)(w \in I(\{v\}))\}
$$

is non-empty.

Moreover, the quotient semigroup

$$
S /(I(K) \phi) \simeq(S-(I(K)) \phi) \cup\{0\}
$$




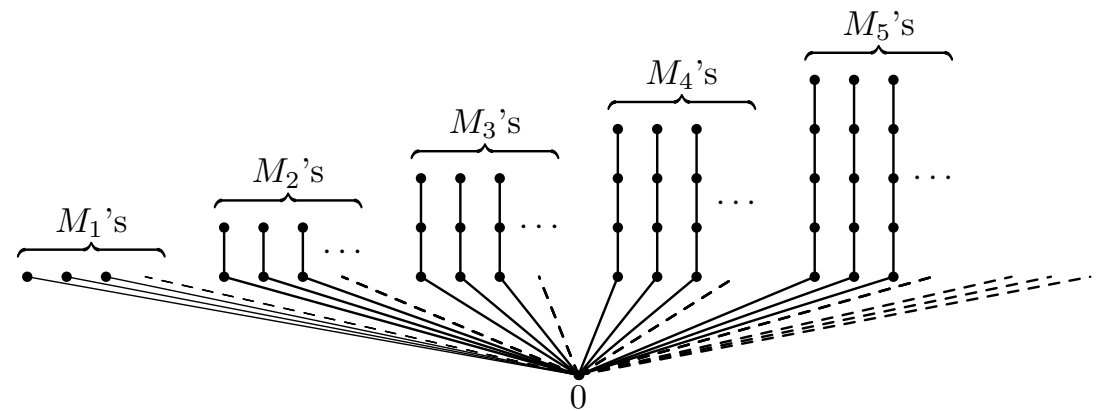

Figure 5: Hasse diagram for the zero-direct union of countably many copies of $(S, \preceq)$.

(where any product in $S$ that lies in $I(K) \phi$ is taken to be 0) is also FApresentable: let $K=(L-I(K)) \cup\{z\}$, where $z$ is a new symbol. It is easy to see that the regularity of $K_{=}$and $K_{\circ}$ follows from that of $L_{=}$and $L_{\circ}$.

The converse does not hold, however: the FA-presentability of a semigroup $T$ does not follow from the FA-presentability of an ideal $I$ of $T$ and the quotient semigroup $T / I$. The counterexample is the semigroup $T$ from Example 7.8, which is not FA-presentable, but which possesses an ideal $I=S_{\beta}$ such that both $I$ and $T / I \simeq S_{\alpha}^{0}$ are FA-presentable.

Furthermore, the class of FA-presentable semigroups is not closed under forming ideals: in Example 10.2, $T$ is a non-FA-presentable ideal of the FApresentable semigroup $S$. Indeed, the following example shows that an ideal $I$ of a semigroup $S$ may not be FA-presentable even when both $S$ and $S / I$ are FA-presentable.

Example 12.1. Let $(S, \preceq)$ be the semilattice from Section 5 . Let $S_{i}$ be isomorphic to $S$ for each $i \in \mathbb{N}$. Then the zero-direct union $T$ of the $S_{i}$ is FA-presentable by Proposition 10.3, and is isomorphic to the semilattice whose Hasse diagram is shown in Figure 5. Let $Y$ be a non-recursively enumerable subset of the natural numbers. Let

$$
I=\{z\} \cup \bigcup_{i \in Y} M_{i},
$$

where the subsets $M_{i}$ lie in $S_{1}$. Then $I$ is an ideal of $S$ that is not FApresentable, being isomorphic to the semigroup $T$ of Example 10.2. Furthermore, the factor semigroup $S / I$ is ismorphic to $S$ itself: to obtain $S / I$ from $I$, one simply deletes one of the [countably many] copies of $M_{i}$ therein for each $i \in Y$; it is clear that the result is isomorphic to $S$.

Acknowledgements. The fourth author would like to thank Hilary Craig for all her help and encouragement. 


\section{References}

[1] H. Ayik and N. Ruškuc. Generators and relations of Rees matrix semigroups. Proc. Edinburgh Math. Soc. (2), 42(3):481-495, 1999.

[2] A. Blumensath. Automatic Structures. Diploma thesis, RWTH Aachen, 1999.

[3] A. Blumensath. Axiomatising tree-interpretable structures. Theory Comput. Syst., 37(1):3-27, 2004. Symposium on Theoretical Aspects of Computer Science (Antibes-Juan les Pins, 2002).

[4] A. Blumensath and E. Grädel. Finite presentations of infinite structures: automata and interpretations. Theory Comput. Syst., 37(6):641$674,2004$.

[5] A. J. Cain. Automatic semigroups and Bruck-Reilly extensions. Submitted

[6] A. J. Cain, G. Oliver, N. Ruškuc, and R. M. Thomas. Automatic presentations for cancellative semigroups. In C. Martín-Vide, H. Fernau, and F. Otto, editors, Language and Automata Theory and Applications: Second International Conference, Tarragona, Spain, March 1319, 2008, number 5196 in Lecture Notes in Computer Science, pages 149-159. Springer, 2008.

[7] A. J. Cain, G. Oliver, N. Ruškuc, and R. M. Thomas. Automatic presentations for semigroups. Inform. and Comput., 2009. To appear.

[8] C. M. Campbell, E. F. Robertson, N. Ruškuc, and R. M. Thomas. Reidemeister-Schreier type rewriting for semigroups. Semigroup Forum, 51(1):47-62, 1995.

[9] C. M. Campbell, E. F. Robertson, N. Ruškuc, and R. M. Thomas. Automatic semigroups. Theoret. Comput. Sci., 250(1-2):365-391, 2001.

[10] C. M. Campbell, E. F. Robertson, N. Ruškuc, and R. M. Thomas. Automatic completely simple semigroups. Acta Math. Hungar., 95(3):201$215,2002$.

[11] D. B. A. Epstein, J. W. Cannon, D. F. Holt, S. V. F. Levy, M. S. Paterson, and W. P. Thurston. Word Processing in Groups. Jones \& Bartlett, Boston, Mass., 1992.

[12] R. Gray and N. Ruškuc. Generators and relations for subsemigroups via boundaries in Cayley graphs. Submitted. 
[13] M. Hoffmann, R. M. Thomas, and N. Ruškuc. Automatic semigroups with subsemigroups of finite Rees index. Internat. J. Algebra Comput., 12(3):463-476, 2002.

[14] J. M. Howie. Fundamentals of Semigroup Theory, volume 12 of London Mathematical Society Monographs (New Series). Clarendon Press, Oxford University Press, New York, 1995.

[15] B. Khoussainov and A. Nerode. Automatic presentations of structures. In D. Leivant, editor, Logic and computational complexity (Indianapolis, IN, 1994), volume 960 of Lecture Notes in Computer Science, pages 367-392. Springer, Berlin, 1995.

[16] G. P. Oliver and R. M. Thomas. Automatic presentations for finitely generated groups. In V. Diekert and B. Durand, editors, 22nd Annual Symposium on Theoretical Aspects of Computer Science (STACS'05), Stuttgart, Germany, volume 3404 of Lecture Notes in Comput. Sci., pages 693-704, Berlin, 2005. Springer.

[17] N. Ruškuc. On large subsemigroups and finiteness conditions of semigroups. Proc. London Math. Soc. (3), 76(2):383-405, 1998.

[18] Sasha Rubin. Automatic Structures. Ph.D. Thesis, Auckland University, 2005. 